\title{
El profesorado y su rol en la formación de los nuevos ciudadanos: desfases entre las comprensiones, las actuaciones y las expectativas*
}

\author{
Teachers and their role in the formation of citizens: Discrepancies between understan- \\ dings, practices and expectations
}

\section{Professorado e seu papel na formação de novos cidadãos: desajustes entre as compre- ensões, as atuações e as expectativas}

\author{
Leonora Reyes J., ${ }^{\mathrm{a}}$ Javier Campos M., ${ }^{\mathrm{b}}$ Luis Osandón M., ${ }^{\mathrm{c}}$ Carlos Muñoz L. ${ }^{\mathrm{d}}$ \\ aUniversidad de Chile. Correo electrónico: leoreyesj@gmail.com \\ bUniversity of Massachusetts Amherst. Correo electrónico: jcampos@educ.umass.edu \\ cUniversidad Academia de Humanismo Cristiano. Correo electrónico: luis.osandon@gmail.com \\ dUniversidad de Concepción. Correo electrónico: carlosem@udec.cl
}

\begin{abstract}
RESUMEN
El presente estudio, a partir de entrevistas semi-estructuradas y registros etnográficos de clases, recoge el análisis de nueve casos de profesores provenientes de tres regiones de Chile que se ocupan de la enseñanza en la asignatura denominada actualmente Historia, Geografía y Ciencias Sociales en el segundo ciclo básico de la educación primaria. El propósito fue conocer cómo comprenden la formación ciudadana desde su ejercicio profesional y cómo la trabajan en sus clases. Los principales resultados demuestran que si bien hay una gran diversidad en el tipo de formación recibida y en la comprensión de las ideas que tienen los docentes sobre qué es formar ciudadanos en la escuela, esto no ocurre del mismo modo en sus clases, donde las estrategias son mucho más homogéneas. Otro resultado es que al momento de planificar sus clases, los docentes se basan más en su experiencia de ejercicio ciudadano antes que en la formación académica recibida en la universidad.
\end{abstract}

Palabras clave: educación, profesión docente, formación ciudadana.

\begin{abstract}
This study, using semi-structured interviews and ethnographic records of classes, collects the analysis of nine teachers from three different regions of Chile who are in charge of the subject denominated History, Geography, and Social Sciences in middle schools. The goal was to understand how they conceive the formation of citizens from their professional performance and how they work with it in their classrooms. Results show that there is a great diversity in the type of training received and the ideas that teachers have about how to form citizens in the school; however, this trend is not replied in same way in their classroom practice where the strategies applied are much more homogeneous. In addition, when teachers plan their lessons, they rely more on their experience as citizens rather than on the academic training received at university.
\end{abstract}

Key words: education, teaching profession, citizens formation.

\section{RESUMO}

Estudo feito a partir de entrevistas semiestruturadas e registros etnográficos de aulas, incluindo a análise de nove casos de professores provenientes de três regiões do Chile, os quais desempenham suas funções profissionais no Segundo Ciclo Básico na disciplina denominada atualmente História, Geografia e Ciências Sociais. Objetivou-se conhecer como eles compreendem a formação cidadã desde seu exercício profissional e como trabalham o tema em suas aulas. Os principais resultados demonstraram que mesmo havendo grande diversidade na formação e na compreensão das ideias que os docentes têm sobre o que é formar cidadãos na escola, tal formação se dá de maneira diferente em suas classes, onde as estratégias são bem mais homogêneas. Outro resultado é que no momento de planejar suas aulas, os docentes se baseiam antes em suas experiências de exercício cidadão e, só depois, na formação acadêmica recebida na faculdade.

Palavras chave: educação, profissão docente, formação cidadã.

* Este artículo forma parte de las conclusiones finales de dos investigaciones financiadas por el Centro de Investigación Avanzada en Educación (CIAE) de la Universidad de Chile. 


\section{INTRODUCCIÓN}

\subsection{EL DESFASE ENTRE POLÍTICAS Y ROL DOCENTE}

Existe un conjunto de factores externos que explican los desfases entre las comprensiones, las actuaciones y las demandas en torno a la formación ciudadana en el sistema escolar. En este sentido, creemos que los cambios que ha vivido la educación escolar contemporánea en el contexto de la globalización y sus consecuentes efectos en las relaciones sociales que se desenvuelven en torno al sistema escolar, inciden de modo importante en esos desfases. Tanto las vivencias y experiencias, como el sentido asignado a la profesionalidad docente en el Chile actual, se encuentran determinadas por una serie de transformaciones ocurridas durante las últimas tres décadas.

Un primer horizonte de análisis se relaciona con la crisis del modelo del capitalismo industrial iniciado hace tres décadas y el surgimiento del nuevo modelo de desarrollo organizado en torno al capital financiero, que desencadenó mutaciones culturales vinculadas a la escuela, el mercado de trabajo, las familias y las comunidades; las que terminaron reconfigurando el rol del trabajo docente en la escuela (Gentili, Apple y da Silva, 1997; Tedesco, 2002; Mejías, 2010). Torres (2000) define este nuevo docente como uno "empobrecido, con menos prestigio, respeto y estatus que el de mitad de siglo, y enfrentado a una tarea mucho más compleja, exigente y vigilada que la de entonces. Las condiciones de trabajo docente se deterioraron y la formación docente ha permanecido aletargada, sin impulso ni condiciones para dar el gran salto requerido".

En la escuela, estas transformaciones se tradujeron en fenómenos como a) la masificación de la escolaridad, b) las transformaciones del mercado de trabajo y de los patrones de construcción de vínculos al interior de las familias y las comunidades, c) el desarrollo explosivo de nuevas "fuentes de socialización", y d) la crisis de las certezas que la escuela parece necesitar, especialmente en relación a la incertidumbre respecto del futuro laboral de los estudiantes, la pérdida de certezas ideológicas y religiosas y la crisis de legitimidad de los ordenamientos políticos y sociales. Todo lo cual hace aparecer al relato escolar como excesivamente rígido y anclado en el pasado ante los ojos de muchos estudiantes y sus familias (Esteve et al. 1997; Hargreaves, 2003, cit. en Cornejo 2009). Esta verdadera revolución económica, social y cultural, constituye el marco ineludible del sistema educativo y de la "cuestión docente" chilena, definida en términos de la reestructuración sufrida por sus salarios y condiciones laborales, así como el bajo impacto de la formación en servicio, la desarticulación entre la formación inicial y continua, entre modalidades y entre niveles, la ausencia de políticas integrales para mejorar la situación de los docentes y la falta de reconocimiento social y valoración de su trabajo (UNESCO, 2012).

En el caso chileno, dos momentos son claves en este proceso. En primer lugar, las profundas transformaciones realizadas por la revolución conservadora ocurrida a partir del golpe militar de 1973, implicaron junto con una brutal represión del movimiento estudiantil y de los sindicatos de trabajadores docentes, la reestructuración global de la relación de estos actores con el Estado. De la mano con la desarticulación del proyecto educacional de la Unidad Popular y el desmantelamiento de la institucionalidad educativa pública, se produjo la reestructuración global del sistema educativo hacia un modelo centrado en el mercado (Ruiz, 2010; PIIE, 1984). 
En segundo lugar, la reforma de 1990, haciendo eco de las transformaciones ocurridas en la década anterior, y en un contexto de transición política hacia la democracia, requirió concretar políticas de reparación en diversos ámbitos, entre ellos el educativo. Para superar los impactos sociales de la descentralización/privatización del sistema escolar y la flexibilización de la relación laboral, que modificó los patrones tradicionales del trabajo docente, se implementaron políticas de desempeño profesional focalizadas mayoritariamente en aspectos contractuales y salariales y no de revalorización de la profesión docente como un todo complejo, íntimamente ligada a su rol social y político.

Una reciente revisión sobre la evolución de la "cuestión docente" en Chile encuentra al menos cuatro grandes transformaciones experimentadas durante las últimas décadas (Cornejo et al. 2008), ellas son: a) la intensificación y estandarización del tiempo de trabajo docente, que implicó el aumento de la cantidad de labores y de responsabilidades asignadas, así como variedad de tareas, en el mismo tiempo de trabajo (OCDE, 2004); b) el abandono de los docentes, en un contexto institucional que fragiliza la labor pedagógica (Cornejo, 2006); c) la culpabilización de los docentes sobre la base de los resultados en pruebas estandarizadas de sus estudiantes (Hargreaves, 2003) y d) el deterioro de los ambientes de trabajo docente (Cornejo, 2009; Valdivia, 2003).

Con todo, desde la década de 2000 se exige que el profesorado responda a los nuevos requerimientos de su profesión en la era de las tecnologías de información y comunicación, la globalización y la sociedad del conocimiento (Brunner, 2003), con "nuevas capacidades profesionales" que lo hagan un "docente efectivo" (Vegas, 2008). Desde el Ministerio de Educación se declara, sin embargo, que "estamos frente a un cuerpo de profesores con carencias y debilidades propias de una etapa de transición, que ha asimilado los cambios a nivel discursivo, pero que no siempre posee las competencias para trasladarlos a la práctica del aula" (Beca et al. 2006: 15). Con mayor énfasis aún, desde algunos sectores del mundo académico se los ha sindicado como el "talón de Aquiles" de las reformas (Bellei, 2001).

Esta visión ha sido reforzada por un contexto laboral desprotegido social y económicamente desde la reforma educativa iniciada en 1980 durante la Dictadura Militar, momento a partir del cual los docentes perdieron sus principales referentes identitarios ligados al Estado de Bienestar: estabilidad (propiedad) del cargo, carrera docente, organizaciones sindicales fuertes y legitimadas socialmente, capacidad de interlocución con el gobierno, etc. Tal como lo ha declarado la Organización para la Cooperación y el Desarrollo Económico (OCDE) en la actualidad “...existe una subestimación del tiempo, los recursos y formación cualitativa de profesores que se requiere para hacer operativas las aspiraciones curriculares en las salas de clases a lo largo del país" (OCDE, 2004: 112-113).

En consecuencia, si el conjunto de transformaciones sociales y culturales ha afectado el trabajo docente en múltiples planos, ya sea en sus capacidades de negociar sus condiciones contractuales del puesto de trabajo, como en el rol profesional y social que debe cumplir en la sociedad contemporánea, la formación de los ciudadanos a través de la escuela se ha visto también afectada. La precarización del puesto de trabajo y los cambios en el entorno sociocultural de la escuela afectan el desempeño profesional de los docentes y, muy sensiblemente, las materias de enseñanza que deben tratar en las aulas. De este modo, conviene examinar los marcos de actuación curricular del profesorado en torno a la formación ciudadana en la actualidad y ver de qué modo ellos están siendo 
comprendidos por los docentes. Así también, la desorientación que se ha producido producto del contexto más arriba descrito, pone en duda la tradición de la "alfabetización constitucional" como clave de la "educación cívica".

\subsection{LA FORMACIÓN CIUDADANA EN EL CURRÍCULUM CHILENO}

El currículum chileno ha sufrido importantes transformaciones en los últimas décadas y existen diferentes descripciones e interpretaciones de sus efectos en el sistema educativo (Magendzo, 2008; Osandón, 2007; Gysling, 2003). La reforma más sustantiva de sus formas y contenidos se produjo a mediados de los años noventa (1996 y 1998 como hitos más relevantes tras la publicación de nuevas normativas curriculares para la enseñanza primaria y secundaria, respectivamente), pero tras casi una década de implementación se elaboraron una serie de ajustes y nuevas herramientas para mejorar sus logros en el aprendizaje de los estudiantes. Así, la mejora más importante al currículum se expresa en los "ajustes curriculares" que afectaron a cinco asignaturas el año 2009. De modo general, en este ajuste se reestructuraron las metas de aprendizaje, junto con la selección y secuencia de los contenidos. Esto resultó especialmente significativo en el ámbito de la enseñanza de la historia y las ciencias sociales. Como consecuencia, entre otras cosas, la asignatura cambió de nombre, desde Historia y Ciencias Sociales a Historia, Geografía y Ciencias Sociales, esta misma denominación pasa a ser única a través de los 12 años de escolaridad (existían tres nombres distintos para distintos niveles de escolaridad) y aparecieron algunos ejes transversales a los niveles de aprendizaje, especialmente hasta finalizar la educación primaria (Osandón, 2013). Del mismo modo, la formación ciudadana pasa a ser un propósito central en la asignatura, entre otras cosas producto de las recomendaciones de la Comisión de Formación Ciudadana impulsada por el Ministerio de Educación unos años antes (Comisión Formación Ciudadana, 2004) y por el avance que supuso la elaboración de los Mapas de Progreso del Aprendizaje que distinguieron tres ejes en la asignatura, uno de ellos denominado Democracia y Desarrollo, el más directamente vinculado a temas de ciudadanía (los otros se refieren a Historia y Geografía respectivamente). ${ }^{1}$

En lo central, el debate en torno a la formación ciudadana en la escuela se ha focalizado en torno a dos propuestas: si esta ha de ser una asignatura particular o bien, como ha sido planteado hasta ahora, un propósito transversal a toda la formación escolar, incluyendo a todas las asignaturas en la tarea de formar al ciudadano.

En el contexto más amplio del debate político y social, las preocupaciones han estado centradas en la utilidad de la transversalidad de la formación ciudadana y su real impacto en la adhesión de las nuevas generaciones a la democracia, entendida principalmente como

\footnotetext{
Al momento de terminar la redacción de este artículo, se ha publicado, no sin tensiones de por medio, una nueva normativa curricular, denominada Bases Curriculares de la Educación Básica (primaria), que fija contenidos y aprendizajes hasta sexto grado. Estas Bases Curriculares destacan la formación ciudadana como un tema central en la asignatura de Historia, Geografía y Ciencias Sociales, pero han surgido múltiples críticas a su formulación, tanto desde el punto de vista técnico como político-ideológico, ello producto de cambios de énfasis en los contenidos. Estos van desde la desaparición del concepto de dictadura militar hasta la comprensión de la ciudadanía como "virtud cívica" en una concepción más bien pasiva del ciudadano, centrando los esfuerzos en la producción de un sujeto subordinado al orden social y, por tanto, poco deliberativo.
} 
el interés de ellas por inscribirse en los registros electorales y votar. ${ }^{2} \mathrm{Al}$ mismo tiempo, algunos intelectuales han enfatizado como propósito central de esa formación ciudadana el logro de la cohesión social, en tanto necesidad de integración de los distintos actores al orden social democrático, construido tras el retorno de la democracia (Cox, Lira y Gazmuri, 2009). Sin embargo, esta lectura parece estrecha a la luz del papel protagónico que el movimiento estudiantil ha asumido en la discusión de un nuevo ordenamiento de los principios que articulan la vida social y política en nuestro país. Entre otras cosas porque los estudiantes han demostrado capacidad para organizarse y deliberar, opacando a una clase política formal que no ha logrado revertir las transformaciones instauradas por la Dictadura Militar que han impedido la democratización efectiva de la sociedad chilena.

Cabe preguntarse entonces por el rol que están jugando los profesores en esa formación de las nuevas generaciones, y como sus estrategias de enseñanza informan estas visiones $\mathrm{u}$ otras tanto en lo que respecta a sus modos de vincular las materias de enseñanza con el ejercicio de la ciudadanía, como sobre sus representaciones del propio rol docente en aquello.

\subsection{LA INVESTIGACIÓN SOBRE LA FORMACIÓN CIUDADANA EN CHILE}

Investigaciones efectuadas en torno a la formación ciudadana en Chile, demuestran que "el espacio y tiempo curricular que ocupan los conocimientos que responden a la racionalidad instrumental, sobrepasan con creces a los conocimientos que apuntan a una racionalidad axiológica y comunicativa, como los que aspiran a formar sujetos de derechos" (Magendzo, 2004: 24). Se ha establecido, además, que este componente de la reforma curricular de los noventa es uno de los menos comprendidos por los docentes y también menos trabajados en las aulas de la Educación Básica (Egaña, 2003). Adicionalmente, el profesorado tiende a entender la formación ciudadana como una instrucción cívica destinada a un ejercicio futuro, coincidiendo con una mayoría de edad y con el aseguramiento de la gobernabilidad; razón por la cual los que realizan en sus clases los docentes está fundamentalmente dirigido a formar gobernados más que gobernantes (Muñoz, Victoriano y Luengo 2011).

Varios son los factores que podrían explicar este fenómeno. Algunos ligados a las vivencias y experiencias del profesorado, mientras que otros están vinculados directamente a su profesionalidad. Entre los primeros se cuentan el temor que aún siente el profesorado al momento de tratar ciertas cuestiones políticas contingentes en sus clases, dado que existen sectores sociales que insisten en imponer su versión sobre determinados hechos, lo que contradice los esfuerzos que realiza la escuela por enfrentar temas contingentes o controversiales (Muñoz, 2008); ello queda refrendado en la investigación de Magendzo y Toledo (2009), donde se evidencia el bajísimo tratamiento de temas como el golpe de estado de 1973 y las violaciones a los derechos humanos posteriores a ese suceso. Por otra parte, conspiran contra un adecuado tratamiento de temas vinculados a la formación ciudadana el excesivo individualismo y competencia existente, la falta de compromiso de la familia y, finalmente, el mal desempeño de algunas autoridades, muchos de cuyos

Cabe señalar que, al momento de escribir este artículo, en Chile la inscripción en los registros electorales era voluntaria y el voto obligatorio solo una vez hecha la inscripción; esto ha envejecido considerablemente el padrón electoral. 
comportamientos actúan como contra ejemplos para lo que procuran promover los docentes en sus clases (Muñoz et al., 2010).

Desde otra perspectiva, referida a la profesionalidad del profesor, es posible relevar la existencia de una formación inicial deficitaria que en general no ha considerado la preparación y actualización sobre ciudadanía como un tema relevante. Esto incide de modo directo en una falta de apropiación curricular sobre la formación ciudadana una vez comenzado el ejercicio profesional de la docencia. Esta falta de apropiación se caracteriza por: el desconocimiento del espacio curricular que ocupa la formación ciudadana en el currículum escolar; una visión inflexible del mismo, que hace al profesorado seguir listados de contenidos sin una articulación más que la disciplinar; una casi exclusiva consideración de contenidos declarativos en sus clases (especialmente conceptuales y factuales), por sobre los procedimentales y valorativos; y finalmente, una manera muy reducida de entender la transversalidad, que configura la formación ciudadana como una cuestión de segundo orden desde el punto de vista curricular, siendo responsabilidad de todos y de nadie (Muñoz et al., 2010).

\section{MARCO TEÓRICO}

\subsection{LAS REPRESENTACIONES SOBRE LOS CONTENIDOS DE LA ENSEÑANZA}

Lo que saben los profesores para hacer sus clases no alude solamente al dominio de contenidos explícitos, supuestamente alineados con lo que el currículum escolar prescribe. Por el contrario, es un saber mucho más amplio que remite a experiencias, formas de ver la realidad y modos de concebir el propio rol en clave de identidad profesional. Lo anterior resulta más relevante aún cuando las expectativas de los actores institucionales juegan un papel importante en la configuración de un espacio dinámico de resignificaciones (Ball y Bowe, 1998; Osandón, 2006). Así, las formas de receptividad de las reformas se pueden expresar como patrones de percepción y reacción construidos a partir de lo que genéricamente conocemos como creencias, vale decir, la acción en aula considera siempre un sustrato "ideológico" que guía la toma de decisiones en el conjunto de la labor profesional del docente. Entonces, se parte de una constatación básica: "El profesor no pone en práctica linealmente un proyecto curricular; por el contrario, lo filtra y redefine en función de demandas que emanan de sus situaciones instructivas, de su conocimiento práctico, de sus estructuras de pensamiento y creencias sobre la educación" (Escudero, 1999: 275). Esto es algo que Thornton (1991) sostuvo hace bastante tiempo, conceptualizando acertadamente al profesor como un gatekeeper o controlador del currículum. Su hipótesis básica para analizar la función controladora del profesorado consiste en considerar que el conocimiento se construye socialmente, es decir, que depende de contextos sociales e históricos. En consecuencia, "para saber cómo actúa el profesorado es necesario averiguar cuáles son sus influencias contextuales y cómo afectan las decisiones que toma" (Thornton, 1991: 44).

\subsection{RELACIONES ENTRE FORMACIÓN Y CREENCIAS EN EL PROFESORADO DE HISTORIA}

Las investigaciones sobre las creencias del profesorado acerca de la enseñanza de la historia y otras ciencias sociales, incluyendo lo que hoy denominamos formación 
ciudadana, tienen una larga trayectoria y sobre ello hay algunas cuestiones necesarias de considerar. Sigrun Gudmundsdottir (1991) estudió el caso de dos profesores experimentados de historia, intentando explicar el conocimiento pedagógico del contenido como un discurso narrativo que le permite a los docentes explicar y explicarse sus propias formas de comprender lo que enseñan. Aquí, las narrativas son entendidas como una forma de conocimiento de la realidad, fuertemente asociadas con los planteamientos de Bruner (2003) acerca del conocimiento narrativo. Dentro de los principales aportes del estudio de Gudmundsdottir se encuentra su demostración de las formas en que se articulan el conocimiento de contenidos disciplinares con el moldeamiento personal del currículo; ello se expresa en estructuras de organización de las clases bajo la modalidad de historias (story). Lo interesante es que es posible encontrar en esas narrativas tanto procesos de organización como de selección y densificación de determinados tópicos, de acuerdo al manejo específico que cada docente tiene de los contenidos, lo que a su vez se vincula con sus creencias y teorías implícitas acerca de los problemas sociales. Así, por ejemplo, es posible observar como un profesor cercano al pacifismo selecciona determinados eventos de la Guerra Civil (o de secesión) americana en función de esos valores. La autora concluye que el conocimiento pedagógico del contenido es en última instancia una forma de organizar narrativas que se transforman en buenas historias para la enseñanza. De esta manera, como lo señala Gimeno (1998), podríamos hablar de una praxis pedagógica, entendida como:

"la interacción entre los significados y usos prácticos del profesor (condicionados por su formación y experiencia, que son las que guían la percepción de la realidad), las condiciones de la práctica en la que ejerce y las nuevas ideas, (...) [elementos que] configuran un campo-problema del que surgen soluciones o acciones del profesor, que son resultantes o compromisos a favor de un extremo u otro de ese triángulo. Es el triángulo de fuerzas de la praxis pedagógica" (Gimeno, 1998: 212).

Esta investigación indaga en esa praxis pedagógica, intentando comprender el modo en que los docentes de Historia, Geografía y Ciencias Sociales reconstruyen los significados de las orientaciones curriculares en torno a la formación ciudadana en el segundo ciclo de la educación primaria (grados 5 a 8).

\subsection{LA COMPRENSIÓN DE LA FORMACIÓN CIUDADANA COMO CONTENIDO DE ENSEÑANZA}

\subsubsection{Minimalismo $v / s$ maximalismo}

Desde el punto de vista curricular y didáctico, la literatura internacional reconoce la existencia de dos grandes enfoques respecto de la relación existente entre la ciudadanía y la educación, esto es: enfoque minimalista y maximalista (Kerr, 2002). El primero es identificado con la educación cívica tradicional y releva conocimientos y contenidos de aprendizaje por sobre el desarrollo de ciertas habilidades y actitudes. De allí que se le considere un enfoque más bien restringido, superficial, excluyente y descontextualizado, aunque naturalmente más fácil de medir y lograr, dada la memorización asociada. El segundo es un enfoque más amplio, que no sólo abarca el conocimiento sino que también la comprensión, la experiencia activa, así como el desarrollo de los valores, disposiciones, habilidades y aptitudes de los estudiantes. Las que son evaluadas en función de problemas reales y actuales de la sociedad (Kerr, 1999). 
Si al enfoque minimalista se le asocia con el concepto de educación cívica, al maximalista se le vincula con el de formación ciudadana, queriendo decir con ello que la vinculación entre la ciudadanía y la educación debe ir más allá de la transmisión de ciertos contenidos y conocimientos, ya sean estos conceptuales o factuales, a saber, desarrollar habilidades y predisposiciones que ayuden al estudiantado a insertarse como genuinos actores de la sociedad de la que forman parte. De allí que mientras el enfoque minimalista busca que el estudiantado aprenda el concepto y la historia de la democracia, el enfoque maximalista aspira a que el estudiantado desarrolle habilidades y disposiciones democráticas. Y mientras el enfoque minimalista pretende enseñar a respetar la ley, el enfoque maximalista aboga no solo por respetarla, sino que también por conocer los mecanismos tendientes a su mejoramiento o abolición.

\subsubsection{Conflicto $v / s$ consenso}

Desde otra perspectiva, la formación ciudadana no puede ser tomada de modo neutral, sus opciones e impactos en la sociedad demarcan un espacio simbólico de alta complejidad desde el punto de vista curricular. Por lo mismo, sostenemos que esa formación debe ser interpretada también a la luz de algunos anclajes conceptuales básicos y propios del debate de la filosofía política y la teoría social.

Al respecto conviene señalar que la vida en sociedad puede ser leída desde a lo menos dos grandes perspectivas, más allá de los matices que se pueden encontrar entre ambas. Por una parte, nos encontramos con la idea de que la sociedad es un todo orgánico, donde actores y procesos se explican por la estabilidad funcional del sistema social (Durkheim, 1995 [1893]). Por otra parte, las teorías sociales que encuentran sus raíces en el marxismo sostienen una perspectiva más confrontacional entre los actores, lo que daría origen a procesos complejos donde la estabilidad y el cambio se conjugan a partir del poder, como eje central de la vida social.

Lo anterior resulta particularmente relevante para comprender los debates en torno a la democracia y los modos de promover su desarrollo. Así, existen posturas que defienden la idea de asegurar la viabilidad de la democracia tomando como base la necesidad de consenso y cohesión de la comunidad política. Pero también es cierto que también es posible encontrar argumentos a favor de una comprensión de la democracia como espacio de antagonismo democrático, como lo sostiene particularmente Mouffe (2003). Es decir, el desarrollo de la democracia no estriba en su estabilidad, sino en la riqueza de sus conflictos productores de transformaciones sociales emancipatorias.

Estas perspectivas tienen una importancia crucial en las orientaciones que puede adoptar la formación ciudadana en la escuela, pues permiten comprender tanto las políticas en torno al tema, así como las prácticas de enseñanza y en su versión más compleja, los resultados formativos de los estudiantes en el mediano plazo. Así, las versiones minimalistas como maximalistas explicadas más arriba deben ser complementadas con estos otros dos conceptos. Ello tiene un potencial heurístico importante en la medida que se pueden localizar las políticas, las prácticas y los resultados, en base a diferentes versiones de la formación ciudadana en la actualidad. Para decirlo en palabras simples, una concepción maximalista de la formación ciudadana puede orientarse tanto a una promoción de la democracia como recurso de estabilización del orden social, o bien como recurso de transformación antagonista del mismo orden social. 


\section{METODOLOGÍA}

\subsection{EL ESTUDIO DE CASOS}

En esta investigación utilizamos la aproximación metodológica del estudio de casos, el cual tiene un carácter empírico, inductivo y comprensivo, es decir, se basa en el razonamiento desde los datos más que en la verificación de hipótesis previamente establecidas (Stake, 1995; García, Gily y Rodríguez, 1996). Trata un fenómeno seleccionado debido a su interés y relevancia para los objetivos de la investigación. En este marco, la presente investigación se constituyó como un estudio de casos múltiples que utilizó información generada a partir de las respuestas que se obtienen de forma parcial en casos analizados individualmente (Yin, 1994). El estudio de casos múltiples permite contrastar distintas fuentes de evidencia "con datos que deben converger en un estilo de triangulación; y, también como resultado, se beneficia del desarrollo previo de proposiciones teóricas que guían la recolección y el análisis de datos" (Yin, 1994: 13). Se sostiene que en comparación a las investigaciones de casos únicos, los resultados son más robustos y convincentes.

\subsection{SELECCIÓN Y CARACTERÍSTICAS DE LA MUESTRA}

La muestra se formó con docentes que imparten el subsector de Historia, Geografía y Ciencias Sociales, en el segundo ciclo básico. Los docentes fueron seleccionados por el equipo de investigación recurriendo a sus contactos previos y a través de recomendaciones de los mismos docentes, usando la técnica de la bola de nieve.

Con estas estrategias fueron seleccionados docentes de las tres principales regiones del país que representaran a las diferentes dependencias administrativas existentes, a saber, municipal, subvencionada y particular.

\subsection{MÉTODO DE RECOLECCIÓN DE DATOS}

La recolección de información sobre los casos incluyó una entrevista semi-estructurada a cada profesor y un registro etnográfico de observación de aula. La pauta de la entrevista semi-estructurada abordó aspectos relacionados con: La trayectoria académica y profesional del entrevistado; la concepción personal de la formación ciudadana; y la representación de la formación ciudadana en el currículum.

\subsection{MÉTODO DE ANÁLISIS DE LOS DATOS}

Los registros y las entrevistas fueron codificados utilizando las dimensiones propuestas por Lam y Kember (2006) para establecer la relación entre las creencias sobre la enseñanza y las aproximaciones a la misma. Entre estas se incluyen: Objetivo de la clase, que incluye las declaraciones y acciones donde se pueda visualizar lo que se espera lograr en la clase; Contenido de la enseñanza, que comprende las declaraciones y acciones explícitas por parte de los docentes sobre los contenidos pertenecientes al currículum explícito y oculto que enseñan; Rol del Maestro que considera las declaraciones y acciones de los docentes vinculadas a lo que debe hacer y cómo se debe comportar un profesor; Rol del estudiante que incluye declaraciones y acciones de los docentes sobre 
lo que debe hacer y cómo se debe comportar un estudiante; Métodos de enseñanza que considera las estrategias pedagógicas o didácticas utilizadas durante la clase; Orientación de la evaluación, que analiza las estrategias e instrumentos de evaluación empleados o reportados durante la observación y la entrevista.

A este conjunto de dimensiones, se incorporó luego de las lecturas iniciales de los datos otras dos propuestas del equipo de investigación, a saber: incidentes críticos, que corresponden a conflictos o acciones vividas como conflicto que ocurren durante las observaciones o se desprenden de los relatos que los maestros realizan y su resolución; y finalmente, la orientación de las preguntas, que incluye las interrogaciones realizadas por el docente durante la observación y la orientación que estas adquieren.

Luego de codificar los datos se realizó un análisis del contenido de las dimensiones, definiendo el análisis de contenido en el marco que propone Maryng, esto es, "como una aproximación empírica, de análisis metodológicamente controlado de textos al interior de sus contextos de comunicación, siguiendo reglas analíticas de contenido y modelos paso a paso, sin cuantificación de por medio" (Mayring, 2000: 1).

A partir del análisis los casos fueron re-organizados y estructurados narrativamente de una manera que permita establecer comparaciones. Así se definieron cinco categorías representativas: Contexto; trayectoria de formación; concepción personal de la formación ciudadana; organización del contenido de la clase; y, finalmente, organización de la estructura interaccional de la clase. Las categorías y su desarrollo son presentadas a continuación.

\section{RESULTADOS}

\subsection{IDEAS DE LOS PROFESORES SOBRE LA ENSEÑANZA PARA FORMAR CIUDADANOS}

Las representaciones de ciudadanía que realizan los docentes entrevistados pueden agruparse, a lo menos, en tres grandes ideas.

Una primera idea da cuenta de una concepción ligada a la formación del individuo, es decir, llegar a ser un "buen ciudadano". Este es definido como quien respeta las normas, los deberes, los derechos y la institucionalidad, como ser un "buen vecino", un "buen alumno" o un "ciudadano para la patria". Una profesora de Valparaíso se refiere como algo positivo el hecho de que los estudiantes sean capaces de "callar frente a una opinión muy diversa con la de él"; adicionalmente señala que tiene que intervenir cuando "está muy efervescente la discusión, se discute a viva voz, fuerte". Dice tener confianza en que los estudiantes "logran entender el propósito de lo que es ser un ciudadano adecuado para la patria" (Caso 6).

Aparece con fuerza la necesidad de preparar y/o entrenar para una inserción "adecuada" (Caso 8) de los estudiantes a la sociedad en que viven, así como involucrarse en la vida cívica para cuando "estén inscritos [en los padrones electorales]". Como lo señala una profesora de un establecimiento de Concepción, "prepararlos para que en el día de mañana, cuando tengan 18 años y estén inscritos en los registros electorales" (Caso 4). En este sentido, el propósito de la vida escolar es más iniciático que terminal:

“... en el segundo ciclo yo estoy como más tirando semillitas, sembrando ciertas nociones antes de tirarlos al gran discurso, los meta relatos” (Caso 2). 
Por otro lado, está la toma de conciencia de que en los deberes y derechos no todos somos iguales, así cada uno adquiere diferentes niveles de responsabilidad en la organización del Estado y la Nación. A este respecto, una profesora pregunta a sus alumnos:

"Qué ha tenido que ocurrir en la sociedad para que lleguemos a tener la sociedad que tenemos actualmente, en la cual todos somos ciudadanos, unos con más o menos derechos, pero todos somos ciudadanos" (Caso 3).

Una segunda idea es aquella relacionada con el desarrollo de los valores del respeto, la tolerancia y la convivencia, entendida como el respeto a la diversidad de credos, etnias, culturas e ideologías. Esta concepción de la diversidad se juega en términos de las múltiples visiones que conviven en la sociedad. A juicio de los y las docentes "todas son respetables" (Caso 8). Se trata de aprender a desarrollar consensos: visión de conjunto. Como lo señala el profesor de un establecimiento de Valparaíso:

“... mientras más diverso mejor. Mientras más debate para mi haya mejor, mientras más posturas de ideas haya mejor... eso o trato de incentivar en todos los planos... los chicos tienen sus opiniones políticas... por último te dirán que un comunista es un infeliz o que pinocho es un asesino, no se, te lo dicen, y ahí yo digo ¿porqué dice usted eso? ¿Qué dice otro? Y ahí vamos construyendo una visión de conjunto..." (Caso 8).

Formarse una opinión y aprender a participar de la sociedad en que se vive, una sociedad ya dada bajo lemas tales como "respeto de las mayorías", "la tolerancia al extranjero" y "la convivencia de todas las visiones" y la "participación en la inscripción electoral" (Caso 9).

Una tercera idea, se relaciona con una concepción de la ciudadanía "procedimental", esto es, la relevancia de los mecanismos de la democracia representativa. Se trata de cambiar la percepción de ciudadanía ligada exclusivamente al terreno de la política parlamentaria ("política fome") y llevarlo a la cotidianeidad:

“... que conozcan algún tribunal, aunque sea por fuera, llevarlos a La Moneda, cuestiones así, llevarlos a los tres poderes del Estado (...) llevarlos a una votación de Junta de Vecinos, qué sé yo, a un Consejo Municipal...” (Caso 8).

El acento es puesto en el procedimiento como una vía para acceder a las finalidades de las actividades relacionadas con generar la posibilidad de comparar distintas realidades y visiones. Es llevar al aula la convivencia, que aprendan a escucharse entre ellos, "practicar con el ejemplo", "poner en práctica el diálogo":

“... si uno como profesor, le muestra que yo lo escucho, que yo le hablo, que me gusta o me interesa tu idea, pero también respeto al otro, entonces ahí yo le puedo exigir al alumno que se respete con los compañeros..." (Caso 5).

También se desarrollan acciones de facto, tales como relevar los apellidos mapuches de los compañeros, de forma de intencionar lo cotidiano como "espacio de aprendizaje". Se arguye al propósito de relacionar la historia personal del niño para darle un sentido a la vida en democracia:

“... podría decir que hay una alumna que tiene apellido Mapuche y que ya tuve una vez. una situación de que un compañero que hizo un comentario que a la compañera le afectó, entonces ya me aproveché de esa situación y estuvimos hablando un poco de las tribus mapuches" (Caso 4). 
Por último, se propone ensayar in situ la vida cívica, como impulsar elecciones de representantes de curso. Una profesora de un establecimiento municipal de Santiago dice que: "ellos siempre se explayan en todo, en todas las cosas y uno les va canalizando las ideas..." (Caso 9).

Entre las metodologías utilizadas se menciona la realización de debates y dramatizaciones. Entre los contenidos de enseñanza se menciona el paso del antiguo al nuevo régimen como una forma de estudiar el ideario liberal asociado a deberes y derechos (Caso 8). Acerca de la auto-percepción del rol docente en la formación ciudadana, se realza la "autonomía" como posibilidad de elegir la vía más "apropiada" para cumplir las finalidades de la formación ciudadana (Casos 1 y 2). Se menciona mucho el uso de "lo cotidiano" para desarrollar ejemplos reconocibles para los niños. En general, este rol es asumido con una relación muy cercana a los niños. Hay referencias en varias entrevistas al desfase entre la formación ciudadana y lo que sucede en la casa. Hay una visión negativa del entorno familiar y sus aportes en esta área "...sus opiniones muy segmentadas, probablemente sus papás..." (Casos 8 y 9).

Por último, en relación a los contenidos, el docente está centrado en la cantidad más que en su calidad (Casos 2 y 8). Siendo especialmente significativa su preocupación por "pasar una materia" o "traspasar" a sus estudiantes una determinada cantidad de contenidos, los que en su mayoría corresponde a aquellos del tipo declarativo (conceptuales y factuales), más que aquellos asociados a una habilidad o predisposición aprovechando un contenido disciplinar, lo que es coincidente con los hallazgos de otras investigaciones mencionadas en nuestro marco teórico.

\subsection{LAS CLASES DE HISTORIA Y LA FORMACIÓN CIUDADANA}

Los docentes suelen señalar que uno de los pocos reductos de autonomía profesional que les va quedando, es la sala de clases. Esta afirmación, que puede ser rápidamente relativizada por el esfuerzo de las políticas públicas en indagar, evaluar y normalizar lo que ahí acontece, la verdad es que sigue teniendo validez en lo fundamental, ello sobre todo por la histórica capacidad de este espacio socioeducativo para neutralizar las iniciativas de cambio e innovación promovidas por las políticas educacionales (Fullan, 1993; Hargreaves, 2003).

Pero hemos dicho que esta investigación no solo indaga las declaraciones de los profesores frente a la formación ciudadana en la asignatura que hoy denominamos Historia, Geografía y Ciencias Sociales, sino que también se propuso reconocer en el espacio de la interacción didáctica y en torno a los contenidos de enseñanza, el modo en que se despliegan los mecanismos tendientes a la formación ciudadana.

Como ya ha quedado dicho en apartados anteriores, la formación ciudadana en el currículum nacional se encuentra transversalizada, lo que representa una oportunidad, así como una gran dificultad, esto es: La posibilidad de que todas las asignaturas contribuyan desde sus saberes específicos a una reflexión sobre las múltiples formas de la ciudadanía y también la invisibilización de aprendizajes específicos sobre este núcleo de la formación escolar.

Señalamos esto pues las observaciones realizadas en este estudio develan estructuras discursivas y modos de interacción en la asignatura de Historia, Geografía y Ciencias Sociales que contribuyen de modo bastante errático a la formación y aprendizaje de cuestiones claves de la formación ciudadana. 
Entenderemos por estructuras discursivas el conjunto de enunciados que los docentes despliegan en el desarrollo de una clase y que refieren centralmente al tratamiento de los contenidos de la asignatura. Por su parte, los modos de interacción refieren a los registros que denotan los mecanismos de gestión del aula en tanto fenómeno de interacción social. Estos últimos refieren fundamentalmente a su regulación. Subyace a estas distinciones una diferenciación entre el conocimiento pedagógico del contenido (Shulman, 1987) y la gestión del aula, desde la perspectiva del currículum oculto (Jackson, 1996; Torres, 2005).

Comenzaremos entonces por exponer algunos rasgos fundamentales de las estructuras discursivas presentes, a través de las observaciones en aula realizadas.

\subsubsection{CIUDADANÍA COMO CONCEPTUALIZACIÓN COGNITIVA}

En el Caso 3, la profesora de la ciudad de Concepción le señala a los estudiantes, a modo de introducción de la actividad de aprendizaje a realizar, que la idea es que no copien del libro la definición de la democracia, sino que "la puedan explicar con sus propias palabras (...) o le encuentran sentido". Sin embargo, la actividad didáctica se desplaza rápidamente a lo que podríamos denominar un análisis con base en el desarrollo de habilidades cognitivas.

"La idea es que ustedes puedan a partir de la conversación grupal sacar las ideas centrales, debilidades de nuestra democracia, fortalezas (...) qué elementos favorece, cierto, están explicitados en nuestra democracia y finalmente que error tiene o tenemos en la convivencia democrática" (Caso 3, Concepción C1).

Lo anterior parece muy plausible dadas las orientaciones curriculares vigentes en la medida que el aprendizaje procedimental, con acento en las habilidades cognitivas, de algún modo conduce las estrategias didácticas al desarrollo de este tipo de aprendizajes, sin embargo, al mismo tiempo, oscurece el propósito original de lo explicitado por la profesora, esto es, llegar a explicar con palabras propias el concepto de democracia y comprender la importancia de la participación "desde pequeños en la organización social" (Caso 3, Concepción B1).

Algo similar acontece con el Caso 4, donde otra profesora en la misma ciudad, enfatizando la idea de la conceptualización descriptiva de algunos rasgos, específicamente de la democracia griega, señala lo siguiente.

"Ya chicos, comenzamos a ver la materia. Vamos a ir leyendo y aclarando los conceptos inmediatamente ¿ya? (...) Pueblo, ¿cierto?, ¿y cracia?, gobierno... entonces "gobierno del pueblo"... sacamos como conclusión que la democracia.... ¿Quiénes eligen a los gobernantes en democracia?, el pueblo ¿cierto?; el pueblo elige a sus gobernantes. Esto es lo que Grecia nos dejó como legado, esto no lo tenemos nosotros, sino que vienen de Grecia y nos deja como una herencia a todo el mundo, y Chile tiene un sistema democrático de gobierno" (Caso 4, Concepción C1).

La tendencia de la docente a anticipar las respuestas, al mismo tiempo que el efecto de contextualización que se da cuando enmarca las respuestas en etiquetas, actúan minimizando la posibilidad de interacción entre los contenidos y las expectativas y experiencias de los estudiantes. En definitiva, creemos que este fragmento representa una de las versiones más clásicas de la enseñanza de la democracia, apelando en lo más sustantivo a la cognición antes que a la experiencia. 


\subsubsection{La ausencia del poder y los procesos}

Una de las cuestiones que más llama la atención de los registros de aula, es lo que podríamos denominar neutralización de los procesos sociales que se tratan a través de la historia de diversas comunidades y tiempos. Dos aspectos resaltan en este fenómeno, de una parte la omisión de los problemas de poder implicados en los conflictos históricos que se revisan en la clase, y de la otra, la pérdida de la noción de proceso, enfatizando el análisis sincrónico antes que el diacrónico.

Un ejemplo de aquello es el tratamiento de la invasión de los persas al territorio de los griegos,

“¿cómo se llamó esa guerra? Defendió su territorio en todo caso Grecia, ¿cierto?, porque el imperio Persa quería atacarlos, quería conquistar su territorio. Se unieron todos... ¿Quiénes se unieron? Todas las polis, sobre todo Esparta y Atenas, ¿cierto?, y ¿cómo se llaman esa guerras? Las Guerras Médicas, ¿cierto? Las Guerras Médicas como una guerra de Grecia con... ¿con quién?...” (Caso 4, Concepción A1).

Es evidente que la intención del docente durante esta intervención es que retengan la triada Persas-Grecia-Guerras Médicas. Sin embargo, en este esfuerzo, se disuelven los problemas culturales, políticos y económicos que involucran el estudio de este proceso histórico. El énfasis en la descripción y la retención de información aislada no permite comprender causas, tensiones, resoluciones más o menos justas de acuerdo a una cierta reflexión crítica de los hechos.

En un sentido similar, otro profesor, tensionado por la necesidad de conceptualizar un proceso, desarrolla la siguiente intervención:

"Profesor: Ya, usted que estás tan motivada ahí la veo, lea la primera pregunta y respuestaAlumna: Describe las características de la cultura germana observada en el texto...Profesor: Muy bien, entonces, estos dos conceptos anótenlos. Entonces, si los bárbaros empezaban a tomar costumbres, los cuadros germanos, costumbres de los romanos, ustedes mismos, como algunos resolvieron, comienzan a tomar o asimilarse mucho al pueblo, ¿no es cierto?, entonces vamos a utilizar este concepto, para resumir eso: romanización, destáquelo ahí con un lápiz rojo..." (Caso 7, Valparaíso A1).

Lo que plantea el docente es un problema interesante: El declive del Imperio Romano conlleva sin duda problemas de mantención de un ideario político y cultural, que se va socavando progresivamente en su última etapa en un proceso de mutuas influencias con los pueblos que iba conquistando. Esto implica una oportunidad para reflexionar sobre los problemas del poder, sobre la lucha por la mantención de una cierta institucionalidad que legitima el dominio de los romanos y, a su vez, el impacto de las prácticas culturales como fenómeno de socavamiento de esas bases políticas. Sin embargo, este espacio de reflexión es obturado por la necesidad de ponerle un nombre al proceso, sea "romanización" o "barbarización", como lo dice a continuación una estudiante en la misma clase.

Por último, una evidencia que contrasta de manera positiva a superar esta ausencia de análisis sobre el poder en el tratamiento de contenidos históricos y sociales es la siguiente intervención, que trata de la estructura de la sociedad colonial en Chile, como contexto que permite comprender el movimiento independentista a comienzos del siglo XIX. Es un abordaje que intenta jugar con la relación pasado-presente. 
"Profesor: Ahora, desde el punto de vista de la sociedad colonial, ¿qué me pueden contar ustedes?

Estudiante: Se conforman por los ricos y por los pobres

Estudiante: Había clases más privilegiadas

Profesor: A ver, en la escala social, ¿Quiénes ocupaban el escalafón importante?

Estudiante: El Rey

Profesor: No, escala social en Chile. Momento... [el profesor pide calma a algunos alumnos que piden la palabra efusivamente] Usted, ¿quiénes ocupaban un lugar de privilegio dentro de la escala social en la sociedad colonial en Chile?

Alumno. Los españoles

Profesor: En las haciendas verdad, en el comercio urbano tenían posibilidades laborales. Ahora claro, evidentemente había indígenas que, producto del tiempo, a lo mejor lograban ascender, pero no era la mayoría. Los mestizos más que nada eran la gente que trabajaba, los que tenían posiciones de prestigio mayor eran peninsulares, españoles o también criollos que eran hijos de los peninsulares. Más abajo, sigamos descendiendo en esta escala social, ¿qué más?...” (Caso 8, Valparaíso D1 y D2).

Hasta aquí, el profesor intenta encuadrar el análisis y la reflexión en la estructura social, caracterizando los espacios sociales de desenvolvimiento de algunos de esos grupos sociales y algunas leves referencias a la movilidad social. En lo que sigue, vienen las referencias al vínculo entre pasado y presente.

"Profesor: Usted, qué me puede indicar, ¿qué diferencias hay en esta sociedad colonial, tradicional, y nuestra sociedad moderna? La de hoy actual, ¿qué diferencias podemos hallar? Alumno: Qué ahora hay más alimentos, como que hacen más alimentos.

Profesor: Este proceso chiquillos implica un acto por el cual los pueblos que habían estado sometidos al poder de la corona española se vuelven el pueblo soberano, ¿qué entendemos por país, por soberanía en el fondo? Por soberanía tenemos que entender un pueblo que tiene la capacidad de crear sus propias leyes, de gobernarse a sí mismo, sin depender de otro estado para que haga o cree sus propias leyes en el territorio. Chile, por ejemplo, actualmente es un país soberano. Tenemos la capacidad de crear nuestras propias leyes, ¿verdad?, dictar nuestras propias normas y para eso hay un cuerpo especializado que es el congreso, el poder legislativo que crea las leyes (...) eso es soberanía, eso es lo que no se tenía antes y es lo que se va a empezar a tener ahora, después del proceso de independencia."(Caso 8, Valparaíso D1 y D2).

Aquí hay una referencia directa a la relación entre pasado y presente y un intento por contextualizar el concepto de soberanía de los estados modernos y de paso, indicar la forma de generación de las leyes en una comunidad. El concepto de transversalización de la formación ciudadana; de este modo, adquiere sentido en la medida que un contenido o tema histórico permite reflexionar sobre el ordenamiento político contemporáneo y comprender las raíces de lo que se tiene en el presente.

En síntesis, los contenidos que se tratan en las clases de historia se encuentran en la mayoría de los casos capturados por la conceptualización de los temas que se trabajan, podríamos decir que esto refiere en lo principal a la idea de retener información clave para poder jugar con la relación descripción-concepto en las evaluaciones que se suelen dar posteriormente, de ahí el énfasis en reiterar y fijar ciertas palabras que resultan claves para una cierta concepción del aprendizaje de la historia. La reflexión, la pregunta sobre el sentido de procesos y hechos históricos, como se puede ver, en general, pierde importancia. 


\subsubsection{Gestionando el aula iformando ciudadanos?}

Un aspecto insoslayable dentro de la perspectiva de la formación ciudadana es el modo concreto en que se verifican las relaciones sociales en el proceso de formación. Como señalamos anteriormente, si bien es muy relevante el contenido de la interacción en el aula, en algunos momentos tanto o más importante es la forma en que el o la docente enmarca las acciones y la forma de los intercambios discursivos sobre el contenido de la clase. Algunas veces, una expresión, una frase, una orden o un llamado de atención trascienden ese contenido y se constituyen en sí mismo en eventos que develan la actitud formativa de él o la docente. En este sentido, lo característico de las observaciones recogidas es la predominancia de la interacción centrada en el control de la conducta.

"Profesor: ¿terminó usted? A trabajar, hay que terminar. [el docente llama por su nombre a un alumno, a quien ha llamado varias veces la atención durante la sesión. Esta amonestación provoca un profundo silencio entre los compañeros] Anotado, es tercera vez que le llamo la atención, ¿quiere que le llame al apoderado para decirle lo mismo que le estoy diciendo yo? Guarde silencio y el resto también" (Caso 8, Valparaíso B1).

Las prácticas de control de la clase de este tipo son frecuentes en las aulas chilenas; sanciones y advertencias forman parte de lo que se suele ver en este tipo de interacciones. Sin embargo, ello resulta más evidente cuando se trata de clases, donde de un modo u otro se intencionan reflexiones sobre el poder, los acuerdos, las reglas de convivencia de las sociedad; esto que hace que resulten ciertamente descontextualizadas. Sin embargo, también se dan con la misma frecuencia, acciones de control del aula que apuntan a motivar un cierto razonamiento sobre las dinámicas grupales coherente con los temas a tratar.

"Profesor: ya chiquillas, la señorita nos va a leer su hipótesis, el resto se calla. Vamos a escuchar (...) cuando un compañero habla todos se callan, la única forma de ser escuchado es que se callen ¿cierto?” (Caso 6, Valparaíso A1).

De este modo, las formas de desarrollar la clase tienen un potencial de incidencia en la formación de disposiciones para el diálogo, la reflexión y el razonamiento colectivo sobre las reglas de la convivencia social en un grado muy importante, tanto por lo que puede impactar en formas de promover la resignación a un rol pasivo, como la posibilidad de alentar la formación de ideas propias. Sobre lo último encontramos pocas evidencias, pues tanto los contenidos como las formas de presentarlos a los estudiantes develan más bien la idea de la transmisión-asimilación.

\section{DISCUSIÓN}

El rol docente ha sufrido un proceso de reconfiguración en los últimos tiempos, este ha venido de la mano con reformas que evalúan el currículum mediante evaluaciones estandarizadas y con regulaciones más fuertes al trabajo en las escuelas. Reformas caracterizadas por ser generadas por expertos, lejos de las aulas y que incluyen a los docentes en procesos de consulta apartándolos de los procesos de decisión. Durante los últimos años estas reformas han incluido la definición de estándares de aprendizaje y su posterior medición en pruebas censales. Estas pruebas más allá de la medición de la calidad de los aprendizajes, tienen una doble función: por un lado regulan a distancia 
el trabajo de los docentes, puesto que, independiente de lo que el docente proponga la prueba mide los contenidos nacionales y solo valida lo que otros han establecido como importante; y por otro lado, estas mediciones hacen a los docentes responsables y muchas veces culpables del resultado de un proceso que trasciende al aula, transformándolos en el chivo expiatorio del sistema educativo.

La formación ciudadana posee un componente ideológico potente sobre lo que implica participar en la sociedad y se juega entre las distintas concepciones sobre el ser ciudadano. Un ciudadano desde una concepción minimalista que busca la estabilidad funcional del sistema social, será entendido como un sujeto que obedece las leyes y se manifiesta a través del voto. El mismo ciudadano, desde una concepción maximalista y crítica, abogará por transformar las mismas leyes a través de la participación y la acción en la sociedad. Estos dos modos de ser ciudadano representan los polos entre los que se mueve un continuo cuyas definiciones son un campo de permanente disputa, desde visiones que apelan a la cohesión social (Cox y Schwartzman, 2009), hasta otras que apuntan al cuestionamiento transformativo del orden social y político (Gentili, 2000), algo que queda reflejado en la prácticas ciudadanas representadas por las movilizaciones sociales recientes en nuestro país.

En este marco los docentes conservan algunos elementos de autonomía, en medio de la aparente ambigüedad que otorga la transversalidad de la formación ciudadana. Esta autonomía se concretiza en una de las ideas que surge desde los resultados de la investigación: la configuración de una tensión muy fuerte entre las expectativas del currículo oficial y el currículo personal del docente. Así, los docentes actúan en el sentido que explicaba Thornton (1991): Integran sus posiciones personales, sus propias narrativas y su visión de lo que significa ser ciudadano en sus prácticas educativas, tornándose efectivamente en un controlador del currículum en la sala de clases.

De esta manera, mientras la formación ciudadana que promueve el currículum oficial en los distintos niveles de escolaridad, ha apuntado en las últimas décadas a generar mayor disposición a participar, a la par de un conocimiento adecuado del ordenamiento jurídico y una comprensión más o menos compleja de cómo transcurre la vida social a través del tiempo, paralelamente, entre los y las docentes, prima una concepción mixta de ciudadanía (maximalista/minimalista), con una clara tendencia a su versión minimalista. Esta entiende la formación ciudadana como aquella que lo lleve a la posibilidad concreta de insertarse a un sistema "ya dado", respetando sus normas, deberes y derechos e institucionalidad, más que a su transformación. En general, los valores asociados a esta concepción de ciudadanía, como la convivencia, el respeto, la diversidad y la tolerancia, obedecen a una comprensión neutral y a-histórica. Así, las evidencias muestran que en general no hay problematización en torno al tema del poder y su compleja configuración a través de las sociedades en el tiempo. Tampoco se los contextualiza históricamente. Todas son respetables, independiente que puedan haber habido situaciones en que algunas miradas se hayan impuestos sobre otras por la fuerza. Este estado de situación de la enseñanza de la historia y las ciencias sociales, sin embargo, también representa una oportunidad para las comunidades y las instituciones que en ellas desarrollan su labor. Esto en la medida que se demuestra que en un tema sensible como la formación ciudadana, los docentes aún manejan márgenes de autonomía, donde sus propias comprensiones y vivencias intervienen el currículo adaptándolo y otorgándole una nueva forma, que es finalmente lo que los estudiantes reciben en sus clases. Desde esta perspectiva, parece necesario incidir 
de modo más directo en una comprensión más compleja de la formación ciudadana por los docentes en la escuela. Esto requiere de una aproximación que abra perspectivas sobre los distintos enfoques de su comprensión y promoción en el proceso formativo.

Por último, cabe hacer notar que los docentes que participan en esta investigación sienten que hacen valer su autonomía cuando pueden, por ejemplo, hacer más "atractiva" la formación ciudadana cuando sacándola del terreno de la política, la acercan a la cotidianeidad de los estudiantes y hacer el ramo "más atractivo" para lograr despertar el interés de sus estudiantes. La "autonomía docente" se asocia entonces a esta posibilidad, más que a la de re-orientar los aprendizajes hacia prácticas transformadoras de la realidad.

\section{CONCLUSIONES}

Los antecedentes presentados corroboran, por una parte, el desfase existente entre las comprensiones de las "políticas públicas" y el "ser docente" y, por otra, que lo que sucede en el aula no se asemeja mucho a lo que manifiestan las directrices y normativas oficiales. Lo que significa -a nuestro entender- que se corrobora lo que muchos estudios sobre el desarrollo curricular señalan, esto es, que no hay un proceso lineal mecánico entre lo indicado por éstas y lo que finalmente acontece en el aula.

Tal cosa se explica, entre otras razones, por la frecuente omisión que se hace de la participación de los docentes en el diseño de estas políticas en materia curricular, en este caso específico, de aquellas que han tenido como objetivo promover la formación ciudadana de los estudiantes. Ello obliga a los docentes a -por ejemplo- hablar de participación sin haber participado en las definiciones centrales de su quehacer profesional. En general solo son requeridos de manera consultiva y parcelada. Lo anterior hace que la formación ciudadana emerja más bien como un discurso, como una consigna, yuxtapuesta, con creencias y representaciones particulares de los problemas y desafíos sociales, que tienden a seguir un modelo peticionista por sobre uno soberano.

Más allá de definiciones compartidas sobre los propósitos finales de este tipo de formación, lo que se constata es un desfase entre lo que la política pública espera y lo que los docentes realizan en sus aulas, cuestión que trasciende los límites de lo que puede realizar la formación inicial docente a la luz de los antecedentes de los docentes entrevistados. A pesar de la diversidad de itinerarios formativos existentes, hay suficiente evidencia que demuestra una marcada homogeneidad en las representaciones, finalidades, tratamiento de contenidos e interacciones de aula. Cada docente actúa de manera individual, con sus propios conceptos y contenidos, con su propia visión de la transversalidad y cotidianeidad, así como con una lógica particular de lo que debe ser su rol profesional y social, pero el resultado final desde la perspectiva de los modos y enfoques de tratamiento son muy similares.

Así, llama la atención que no se "haya puesto en funcionamiento" de parte de los profesores estrategia alguna para enfrentar este nuevo requerimiento curricular. Así tampoco existen mayores indicios de una reflexión profunda y crítica sobre su práctica, sus representaciones, finalidades, contenidos y formas de interactuar y enseñar.

Por último, pensando proyectivamente los resultados de esta investigación, un desafío para los académicos de las escuelas de pedagogía se relaciona con fortalecer la formación en este ámbito, pero no en clave de rudimentos de derecho o de ciencia política, sino 
a través de una formación seria y rigurosa en torno al debate sobre la ciudadanía en el mundo contemporáneo, sobre sus implicancias, éticas y políticas en el plano pedagógico y en torno a sus diversas alternativas de concreción en el aula a través de las asignaturas escolares. Lo anterior debiera redundar en mejores capacidades reflexivas y creativas de los estudiantes de pedagogía, de manera que estos puedan apropiarse activamente del currículo utilizando de manera consciente y reflexiva su experiencia. Por otro lado, estos resultados llevan a poner atención sobre la necesidad de preservar, reconstruir o potenciar los espacios e intersticios de relativa autonomía de los docentes en los establecimientos escolares de manera de poder decidir la posición desde la cual ejercerán su labor pedagógica en la formación de los ciudadanos.

\section{REFERENCIAS BIBLIOGRÁFICAS}

Ball, S. y R. Bowe (1998). El currículum nacional y su "puesta en práctica". El papel de los departamentos de materias o asignaturas. Revista de Estudios del Currículum, vol. 1, n. 2, 105-131.

Beca, C.; P. Montt; C. Sotomayor, J. García-Huidobro \& H. Walker (2006). Docentes para el nuevo siglo: hacia una política de desarrollo profesional docente. Santiago: MINEDUC.

Bellei, C. (2001). ¿Ha tenido impacto la reforma educativa chilena? En C. Cox (Ed.), Políticas educacionales en el cambio de siglo. La reforma del sistema escolar de Chile (pp. 125-209). Santiago: Editorial Universitaria.

Bruner, J. (2003). La fábrica de historias. Derecho, literatura, vida. Buenos Aires: FCE.

Brunner, J. J. (2003). Educación e Internet ¿La próxima revolución? Santiago: Fondo de Cultura Económica.

COMISIÓN FORMACIÓN CIUDADANA (2004). Informe Comisión Formación Ciudadana. Santiago de Chile: MINEDUC.

Cornejo, R. (2006). El experimento educativo chileno 20 años después: una mirada crítica a los logros y falencias del sistema escolar. Revista Electrónica Iberoamericana sobre Calidad, Eficacia y Cambio en Educación, vol. 4, n. 1, 118-129.

Cornejo, R. y Reyes, L. (2008). Chile: experiencias organizacionales y acción colectiva de profesores. Colección Libros FLAPE 23: disponible en: http://www.foro-latino.org/flape/producciones/ coleccion_Flape_08/23\%20Chile\%20Docente.pdf

Cornejo, R. (2009). Condiciones de trabajo y bienestar/malestar docente en profesores de enseñanza media de Santiago de Chile. Educación y Sociedad, vol. 30, n. 107, 409-426.

Cox, C.; R. Lira y R. Gazmuri (2009). Currículos escolares y sus orientaciones sobre historia, sociedad y política: significados para la cohesión social en Latinoamérica. En Cristián Cox y Simon Schwartzman (Eds.), Las políticas educativas y la cohesión social en América Latina (pp. 235-292). Santiago: Uqbar editores.

Durkheim, E. (1995[1893]). La división del trabajo social. Madrid: Akal.

Egaña, L. (2003). Reforma Educativa y Objetivos Fundamentales Transversales. Los dilemas de la innovación. Santiago: PIIE.

Escudero, J. M. (ed.) (1999). Diseño, desarrollo e innovación del currículum. Madrid: Síntesis.

Esteve, J., Franco, S. y Vera, J. (1997). Los profesores ante el cambio social. Anthropos: Madrid.

Fullan, M. (1993). Change Forces. Probing the depths of educational reform. UK/USA: Falmer Press.

García, E., Gil, J. y Rodríguez, G. (1996). Metodología de la Investigación Cualitativa. Málaga: Aljibe.

Gentili, P. (Comp.) (2000). Códigos para la ciudadanía. La formación ética cómo práctica de libertad. Buenos Aires: Santillana. 
Gentile, P.; M. Apple y T. T. da Silva (1997). Cultura, Política y Currículo. Ensayos sobre la crisis de la escuela pública. Buenos Aires: Losada.

Gimeno, J. (1998). El currículum: una reflexión sobre la práctica. Morata, Madrid.

Gudmundsdottir, S. (1991). Pedagogical models of subject matter. En J. E. Brophy (Ed.), Advances in research on teaching: teachers subject matter knowledge and classroom instructions. Greenwisch, CT: JAI Press.

Gysling, J. (2003).Reforma curricular: itinerario de una transformación cultural. En C. Cox (Ed.), Políticas educacionales en el cambio de siglo: La reforma del sistema escolar de Chile. Santiago de Chile: Editorial Universitaria.

Hargreaves, A. (2003). Profesorado, cultura y postmodernidad. Madrid: Morata.

Jackson, Ph. (1996). La vida en las aulas. Madrid: Morata.

Kerr, D. (1999). Citizenship Education: an International Comparison. London: National Foundation for Educational Research in England and Wales NFER/ Qualifications and Currículum Authority in England QCA.

Kerr, D. (2002). An international review of citizenship in the currículum. En G. Steiner-Khamsi, J. Torney-Purta y J. Schwille (Eds.), New paradigms and Recurring Paradoxes in Education for Citizenship: an international comparison. UK: Elsevier Science Ltd.

Lam, B. \& Kember, D. (2006). The relationship between conceptions of teaching and approaches to teaching. Teachers and Teaching: Theory and Practice, vol. 12, n. 6, 693-713. doi:10.1080/13540600601029744

Mayring, P. (2000). Qualitative content analysis. Qualitative Sozialforschung/Forum: Qualitative, vol. 1, n. 2. Retrieved from http://www.utsc.utoronto.ca/ kmacd/IDSC10/Readings/text analysis/ CA.pdf

Magendzo, A. (2008). Dilemas del currículum y la pedagogía: Analizando la reforma curricular desde una perspectiva crítica. Santiago: LOM.

Magendzo, A (2004). De miradas y mensajes a la educación en derechos humanos. Santiago: LOM.

Magendzo, A., y Toledo, M. I. (2009). Moral dilemmas in teaching recent history related to the violation of human rights in Chile. Journal of Moral Education, vol. 38, n. 4, 445-465.

Mejías, M. R. (2010). Reconfiguración de la pedagogía, pedagogías críticas y movimientos pedagógicos en el contexto educativo actual. Ponencia presentada al Encuentro Nacional de Movimiento Pedagógico, Colegio de Profesores de Chile, Santiago de Chile, 13 al 15 de enero.

Mouffe, Ch. (2003). La paradoja democrática. Gedisa: Barcelona.

Muñoz, C.; R. Victoriano y H. Luengo (2011).La compleja tarea de formar ciudadanos. Conocimientos, habilidades y disposiciones promovidas en Lenguaje y Comunicación y Estudio y Comprensión de la Sociedad en la EGB. En MINEDUC (Comp.), Evidencias para políticas públicas en Educación. Selección de investigaciones Tercer Concurso FONIDE (pp. 55-93). Santiago: Centro de Estudios MINEDUC.

Muñoz, C.; R. Victoriano; H. Luengo; N. Naíl. y N. Ansorena (2010). El desafío de la Formación Ciudadana. Principios para un modelo de integración curricular en Lenguaje y Comunicación y Estudio y Comprensión de la Sociedad para la EGB. Concepción: Ediciones Universidad de Concepción, Trama Impresores.

Muñoz, C. (2008). Desarrollar competencias. Un desafío de los docentes de Primaria. Revista AULA, Innovación Educativa, n. 170. En línea; disponible en: http://phobos.xtec.cat/crp-santceloni/ nous_currículums/nous_currículums/desarrollar\%20competencias.pdf

OCDE (2004). Organización para la cooperación y el desarrollo económico. Francia: Centro para la cooperación con países no miembros.

Osandón, L. (2006). Los docentes frente a una innovación curricular. Experiencias de resignificación de los Programas de Estudio en Historia y Ciencias Sociales. Tesis de Doctorado en Ciencias de la Educación. Santiago: Pontificia Universidad Católica de Chile. 
Osandón, L. (2007). Lecciones de una década de reforma al currículum: ¿hemos aprendido a trabajar con el profesorado y las instituciones educativas? Revista Foro Educacional, n. 12, 129-157.

Osandón, L. (2013). Ajustes curriculares a la enseñanza de la historia, trayectorias y proyecciones. En I. Muñoz D. y L. Osandón M. (Comps.), La didáctica de la historia y la formación de ciudadanos en el mundo actual. Santiago: DIBAM.

PIIE (1984). Transformaciones educacionales bajo el régimen militar. Santiago: PIIE.

Ruiz, C. (2010). De la República al mercado. Ideas educacionales y política en Chile. Santiago: LOM.

Sennett, R. (1998). La corrosión del carácter. Consecuencias personales del trabajo en el nuevo capitalismo. Barcelona: Anagrama.

Shulman S. (1987). Knowledge and teaching: foundations of the new reforms. Harvard Educational Review, vol. 57, n. 1, 1-22.

Stake, R. E. (1995). Análisis e interpretación en Investigación de estudio de casos. España: Editorial Morata.

Tedesco, J.C. y E. Tenti (2002). O Desempenho dos professores na América Latina e Caribe: Novas Prioridades. Brasilia: Conferencia Regional, BID/UNESCO/MINISTÉRIO DA EDUCAÇAO.

Thornton, S. (1991) Teacher as curricular-instructional gatekeeper in Social Studies. Shaver, J. P. (edit.), Handbook of Research on Social Studies teaching an learning. A project of The National Council for the Social Studies (pp. 237-248). New York: Macmillan.

Torres, J. (2005). El currículum oculto. Madrid: Morata.

Torres, R. M. (2000). De agentes de la reforma a sujetos del cambio: la encrucijada docente en América Latina. Perspectivas, vol. XXX, n. 2, 1-21.

UNESCO (2001). Antecedentes y criterios para la elaboración de políticas docentes en América Latina. Santiago de Chile: UNESCO.

Valdivia, G.; C. Avendaño.; G. Bastías.; N. Milicic; A. Morales y J. Scharager (2003). Estudio de la salud laboral de los profesores en Chile. Santiago: Pontificia Universidad Católica de Chile. Escuelas de Medicina y Psicología. Facultad de Medicina y Ciencias Sociales.

Vegas, E. (2008). ¿Cómo mejorar las políticas de desarrollo profesional docente a fin de atraer, perfeccionar y retener profesores efectivos? En C. Bellei, D. Contreras, J. P. Valenzuela (Eds.), La agenda pendiente en educación (pp. 48-78). Santiago: Universidad de Chile.

Yin, R. K. (1994). Case Study Research: Design and Methods. CA: Sage. 
